\title{
Automatic View Planning for Cardiac MRI Acquisition
}

\author{
Xiaoguang Lu ${ }^{1, \star}$, Marie-Pierre Jolly ${ }^{1}$, Bogdan Georgescu ${ }^{1}$, Carmel Hayes ${ }^{2}$, \\ Peter Speier ${ }^{2}$, Michaela Schmidt ${ }^{2}$, Xiaoming $\mathrm{Bi}^{3}$, Randall Kroeker ${ }^{4}$, \\ Dorin Comaniciu ${ }^{1}$, Peter Kellman ${ }^{5}$, Edgar Mueller ${ }^{2}$, and Jens Guehring ${ }^{2}$ \\ ${ }^{1}$ Image Analytics and Informatics, Siemens Corporate Research, Princeton NJ, USA \\ xiaoguang.lu@siemens.com \\ 2 Siemens AG, Healthcare Sector, H IM MR PLM-AW CARD, Erlangan, Germany \\ ${ }^{3}$ Siemens Medical Solutions USA, Chicago IL, USA \\ ${ }^{4}$ Siemens Medical Solutions Canada, Winnipeg MB, Canada \\ ${ }^{5}$ National Institutes of Health, Bethesda MD, USA
}

\begin{abstract}
Conventional cardiac MRI acquisition involves a multi-step approach, requiring a few double-oblique localizers in order to locate the heart and prescribe long- and short-axis views of the heart. This approach is operator-dependent and time-consuming. We propose a new approach to automating and accelerating the acquisition process to improve the clinical workflow. We capture a highly accelerated static 3D full-chest volume through parallel imaging within one breath-hold. The left ventricle is localized and segmented, including left ventricle outflow tract. A number of cardiac landmarks are then detected to anchor the cardiac chambers and calculate standard 2-, 3-, and 4-chamber long-axis views along with a short-axis stack. Learning-based algorithms are applied to anatomy segmentation and anchor detection. The proposed algorithm is evaluated on 173 localizer acquisitions. The entire view planning is fully automatic and takes less than 10 seconds in our experiments.
\end{abstract}

\section{Introduction}

Accurate morphological and functional measurements of the heart are essential in clinical applications for diagnosis, prognostic, and therapeutic decisions. Magnetic resonance imaging (MRI) allows morphological characterization of heart structures with precision. For the past decade, there has been a great deal of efforts focused on automatic cardiac segmentation [5]12], however, less attention has been paid to automatic and fast planning for cardiac MRI acquisition 7846 , which still remains challenging in clinical practice.

During conventional cardiac MRI acquisition, anchoring the heart is typically performed using a multi-step approach involving the acquisition of doubleoblique slices in order to localize the long and short-axis of the heart. Based on those localizers the standard heart views (e.g, 2-chamber, 3-chamber, 4-chamber

^ Corresponding author.

G. Fichtinger, A. Martel, and T. Peters (Eds.): MICCAI 2011, Part III, LNCS 6893, pp. 479-486, 2011.

(C) Springer-Verlag Berlin Heidelberg 2011 
and short-axis views from base to apex) are planned. This approach is operatordependent and time consuming. For example, a typical protocol starts with capturing coronal localizers, followed by multi-slice localizer at different orientations in order to align the left ventricle (LV) with the isocenter of the scanner; then one vertical long-axis localizer is planned on transversal slice and one horizontal long-axis localizer is planned on vertical long-axis view, from which a few shortaxis views are planned; based on the short-axis views, 2-, 3-, and 4-chamber views are planned; and finally the short axis stack is planned on the 4-chamber view. It requires detailed knowledge of the heart for operators to plan the views at every step during this long process, while the patient is in the scanner.

As parallel MR imaging techniques rapidly advance, single breath-hold acquisitions with full chest coverage become feasible. Instead of acquiring a series of 2D localizer slices with operator interventions, a direct approach to prescribing the clinically relevant slices in a fully automated fashion from a single acquisition would be highly beneficial. However, this is a challenging task in order to be clinically viable because it requires a robust approach to differentiating the heart from other complex anatomical structures in a full-chest volume, accounting for large variations across populations, and with quick response time.

Lelieveldt et al. 78 proposed fuzzy implicit surface templates to model thoracic anatomies and automatically estimate the LV orientation for view planning. The method was evaluated on 20 volumes and planning was for short-axis stack only. Danilouchkine et al. 4, quantitatively evaluated the difference of clinical meansurements derived from acquisitions between manual and automatic planning on 10 healthy volunteers, concluding that automated CMR planning methods could provide accurate measurements of LV dimensions in normal subjects. In addition to the short-axis stack, long-axis images also provides clinical values, such as through-plane motion analysis [2]. Jackson et al. 6] proposed a semiautomatic approach for planning short-axis stack, and two long-axis views, i.e., HLA (4-chamber view) and VLA (2-chamber view). User interaction is needed for chamber segmentations, which were based on an EM algorithm. The approach was evaluated on 12 volunteers.

We propose a fully automatic algorithm to prescribe short-axis stack and standard long-axis view, including 2-, 3-, and 4-chamber views, from a single 3D MR volume for cardiac MRI acquisition. Our approach to improving the clinical workflow is based on machine learning based methods to localize and delineate cardiac anatomies in a $3 \mathrm{D}$ volume, and detect a set of cardiac landmarks to anchor chambers in order to prescribe the views. The proposed approach is evaluated on 173 localizer volumes from 100 subjects.

\section{Methodology}

\subsection{Overall Workflow}

Our view planning workflow for cardiac MRI acquisition as shown in Fig. 1 contains the following steps: 1) 3D static volume acquisition; 2) LV localization (pose 


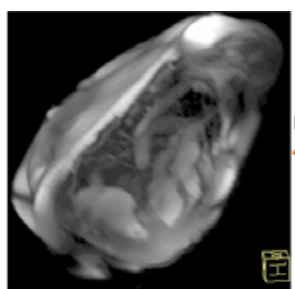

(1) Full-chest volume
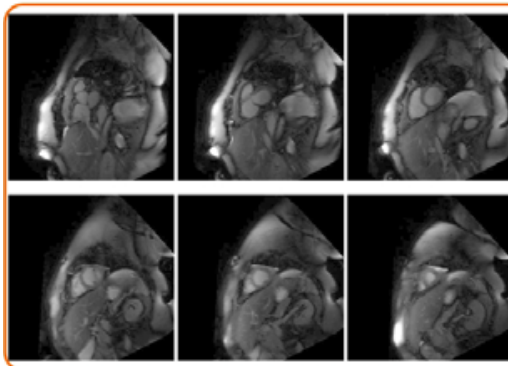

Short axis stack

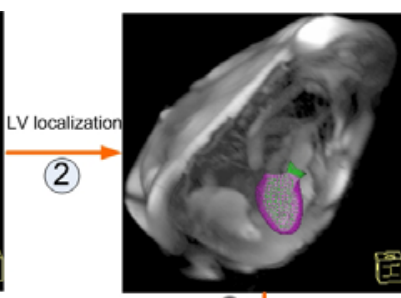

(3) 1
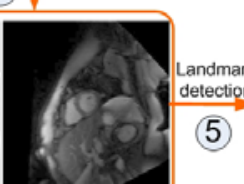

(4)

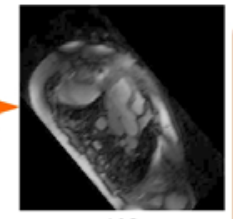

$\mathrm{A} 3 \mathrm{C}$

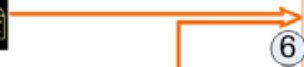

6

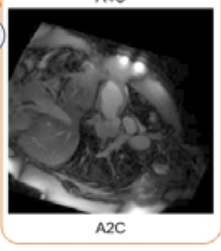

Fig. 1. System workflow, Numbers indicate the steps illustrated in Sec. 2.1

estimation) and delineation (boundary extraction); 3) short axis stack prescription; 4) 3-chamber view calculation; 5) landmark detection in mid-ventricular slice(s);6) 4-chamber and 2-chamber view calculations.

For LV delineation and landmark detection, we adapted the basic approaches presented in [12] and 9] due to computational efficiency and accuracy. For completeness, we briefly describe the methods in our application context.

A mesh representation of the anatomical shape is created using a database of $3 \mathrm{D}$ volumes that are manually delineated. For left ventricle, this anatomically accurate model is composed of LV endocardium, LV epicardium, and LVOT. The LV model is a triangular mesh as shown Fig. 2. It is used to fit to a given $3 \mathrm{D}$ cardiac volume to delineate corresponding anatomical structures. Including LVOT into our workflow helps anchor the 3 -chamber view.

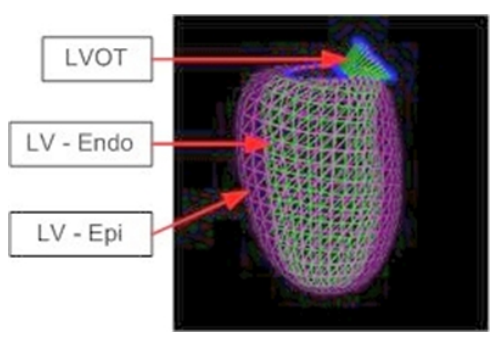

Fig. 2. Triangular mesh model for left ventricle and LV outflow tract (LVOT) 


\subsection{Localization and Delineation}

The LV pose (i.e., positions, orientations, and scales) in a 3D volume is first estimated at the LV localization stage. At the subsequent delineation stage, local deformations of the model with the estimated pose are applied in order to fit the model to LV boundaries.

Learning-based object detection approaches have been demonstrated successful in many applications [11. Learning-based methods trained on expert annotations are appropriate to handle complex appearance and heterogeneous characteristics of anatomical features in medical images, as the complex prior knowledge is implicitly encoded.

In order to estimate the LV pose, we need to solve for the nine-parameter space, including three translations, three orientations, and three scales. We design a series of detectors that estimate parameters at a number of sequential stages in the order of complexity, i.e., translation, orientation, and scale. Multiple hypotheses are maintained between stages, which quickly removes false hypotheses at the earlier stages while propagating the right hypothesis to the final stage. Only one hypothesis is selected as the final detection result.

A large database is collected with LV annotated. For each detector, a set of positive and negative samples are extracted from the annotated database with positive samples as the true targets (close to the ground truth) and negative samples as others (far away from the ground truth). The detection task is a binary classification. Our detectors to estimate the LV pose and extract LV boundaries are constructed using probabilistic boosting trees (PBT) [10, which select a set of discriminative features that are used to distinguish the positive samples from negatives from a large pool of features. The classifier is a tree-based structure with which the posterior probabilities of the presence of the object of interest are calculated from given data. The nodes in the tree are constructed by a nonlinear combination of simple classifiers using boosting techniques. For the detector at the translation stage, we choose 3D Haar wavelet-like features, which are calculated efficiently using integral image-based techniques. For the detectors at the orientation and scale search stages, steerable features [12 are applied, whose computation does not require volume rotation and re-scaling, which are computationally expensive, especially when the hypothesis search space is large. The boundary detectors also use steerable features.

With the model pose estimated, we align the mean shape (average model of all annotations) with data to get an initial estimate of the object shape. To capture the true anatomical morphology of the LV shape, we deform the mean LV model by searching the boundary for each vertex of the model. The boundary hypotheses are taken along the normal directions at each vertex of the mean model. Detection is achieved using a boundary detector trained by PBT with steerable features. The detected boundaries are constrained by projecting the detected model onto a shape subspace obtained by the annotated dataset, which was constructed using principal component analysis [3. As a result, the mesh model is fitted to given volumetric data in alignment with the LV. 


\subsection{View Planning}

With the left ventricle delineated, a few cardiac anchors are computed. In our mesh model, the LV base and apex have corresponding vertices. The LV base center is calculated as the centroid of the vertices on the LV base. The LV long axis is calculated by connecting the LV base center and the apex, which is also used to plan the short-axis stack. The method of fitting a line across centers of all short axis MPRs as shown in [7] and 6] is also applicable. The entire short axis stack can then be planned from base to apex. To plan the 2- and 4- chamber views, we reconstruct a mid-ventricular short axis slice (MPR) from the short axis stack, and apply the landmark detection algorithm proposed in 9 . to localize the anterior and inferior RV insertion points. The 2-chamber view is calculated to be in parallel to the line connecting the two RV insertion points and cross the LV blood pool center that lies on the short-axis MPR used for landmark detection, which is obtained through the detected LV mesh. The 4chamber view is computed to cross the LV blood pool center and the RV lateral point (the green landmark in Fig. 1). Fig. 1 presents an example of all planned views.

\section{$3 \quad$ Experiments}

A 3D full-chest MR scan was obtained through parallel imaging within a single breath-hold. A single volume was acquired at mid-diastole using an ECG gated segmented acquisition with T2-prepared SSFP readout with chemical shift fat suppression. Typical protocol parameters are: 400x400x220 $\mathrm{mm}^{3}$ FOV prescribed as a coronal slab with matrix of $256 \times 202 \times 44$, corresponding to $1.6 \times 2 \times 5 \mathrm{~mm}^{3}$ resolution, interpolated to $2.5 \mathrm{~mm}$ slices. Images are acquired on $1.5 \mathrm{~T}$ Siemens MAGNETOM Avanto/Espree with a 32 channel coil enabling parallel imaging with acceleration rate $6=3 \times 2$ ( $\mathrm{PE}$ in LR direction $\mathrm{x}$ PAR in AP direction) and $3 / 4$ partial Fourier in PAR dimension. Breath-hold duration is typically less than 20s with all PE lines acquired in a single shot per heartbeat. 88 volumes from 77 patients were acquired under this protocol, which we called the baseline protocol. In addition, a few experimental localizer protocols were applied to investigate the image variations by modifying acquisition parameters such as the number of breathholds, the number of slices per heart beat, real-time acquisition, etc., resulting in additional 85 volumes from a combination of 23 volunteers and patients. In total, 173 localizer volumes from 100 subjects were collected. Examples acquired with different protocols are provided in Fig. 3 .

For each volume, the left ventricle was manually delineated using triangular mesh representations as ground truth, including endocardium, epicardium, and LVOT boundaries. The standard 2-, 3-, 4-chamber views were also manually identified by visually navigating the volume in $3 \mathrm{D}$, with each view recorded by a combination of a plane normal vector and an anchor on the plane. The LV long axis groundtruth is calculated as a vector from the LV apex pointing toward LV base center (the centroid of mesh points in the mitral plane), which was used as the normal direction of the short-axis stack. 


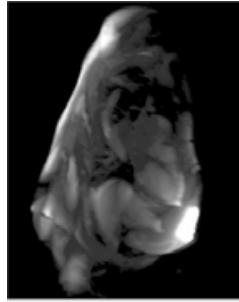

(a)

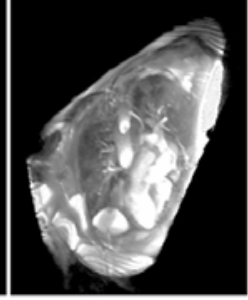

(b)

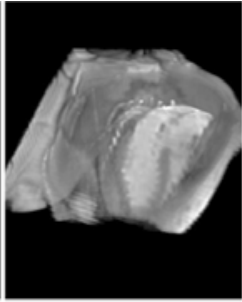

(c)

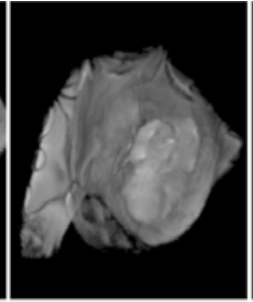

(d)

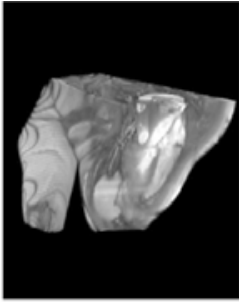

(e)

Fig. 3. Examples of volumes captured with different protocols in our database. (a)-(b) Acquisitions using the baseline protocol protocol. (d)-(e) Acquisitions with the variants of the baseline protocol.

A 4-fold cross-validation scheme was applied for evaluation. The entire 173 volumes were randomly partitioned into four quarters. No volumes from the same subject were partitioned into different quarters. For each fold evaluation, three quarters were combined for training and the remaining one quarter was used as unseen data for testing. This procedure was repeated four times so that each volume has been used once for testing. To evaluate the LV delineation results, for each fitted mesh, the distance from each vertex to the groundtruth mesh (manual annotation) was computed (so called point-to-mesh distance). The average of distances from all vertices of the fitted mesh was used as the measurement. The mean, standard deviation, and median values of this distance measurement are $4.96 \mathrm{~mm}, 10.35 \mathrm{~mm}$, and $2.51 \mathrm{~mm}$, respectively, which indicate a good delineation and anchoring for the subsequent view calculation. Large standard deviations are due to detection outliers.

Fully automated view planning examples are provided in Fig. 4, For short-axis stack planning evaluation: the LV long axis (i.e., the normal of the prescribed short-axis stack) is used to evaluate short-axis stack axis direction estimation accuracy, which is calculated as the angle between the estimated LV long axis and the annotated ground truth; to evaluate the coverage of the prescribed short-axis stack, the groundtruth LV base and LV apex are projected onto the estimated LV long axis, then the distances from the projected groundtruth to the automatically estimated base and apex positions are computed. For longaxis view planning evaluation: each estimated view is represented by its normal vector and an anchor on the plane; the angle difference is calculated between the normal vector and the ground truth normal direction; the anchor position difference from the groundtruth is computed as the point-to-plane distance from the anchor on the detected view to the annotated groundtruth view plane; the anchor on the detected view is the LV blood pool center on the prescribed midventricle slice (see Sec. 2.3) for landmark detection. Performance is summarized in Table 1. Large errors are mostly due to image artifact and poor image quality such as the examples shown in Figs. 3(c) and (d).

On average, it took about 5 seconds to localize and delineate the LV from a single full-chest volume (e.g, $256 \times 256 \times 112$ ), and about half a second to detect landmarks from a mid-ventriclar slice. The entire LV anchoring and view planning process took about 7 seconds on an Intel core-duo $2.66 \mathrm{GHz} \mathrm{CPU}$. 


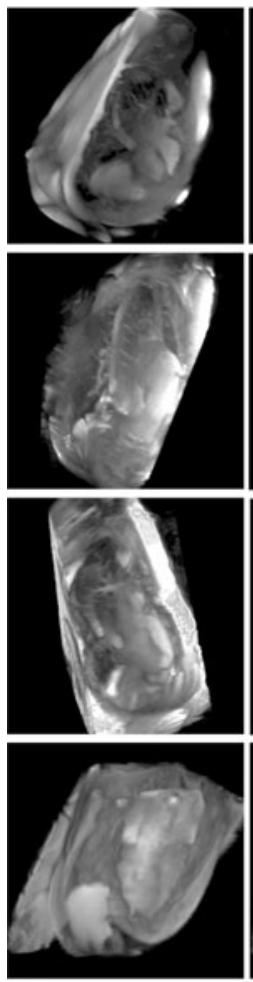

(a)
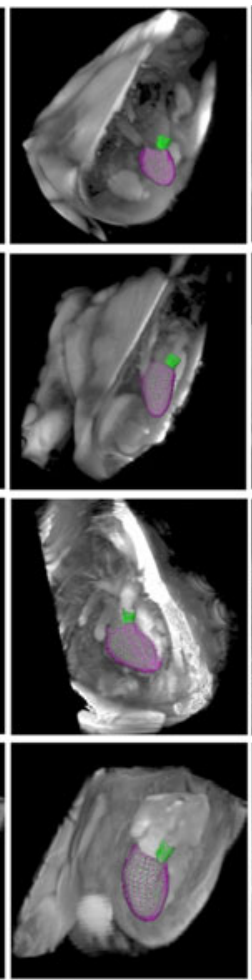

(b)
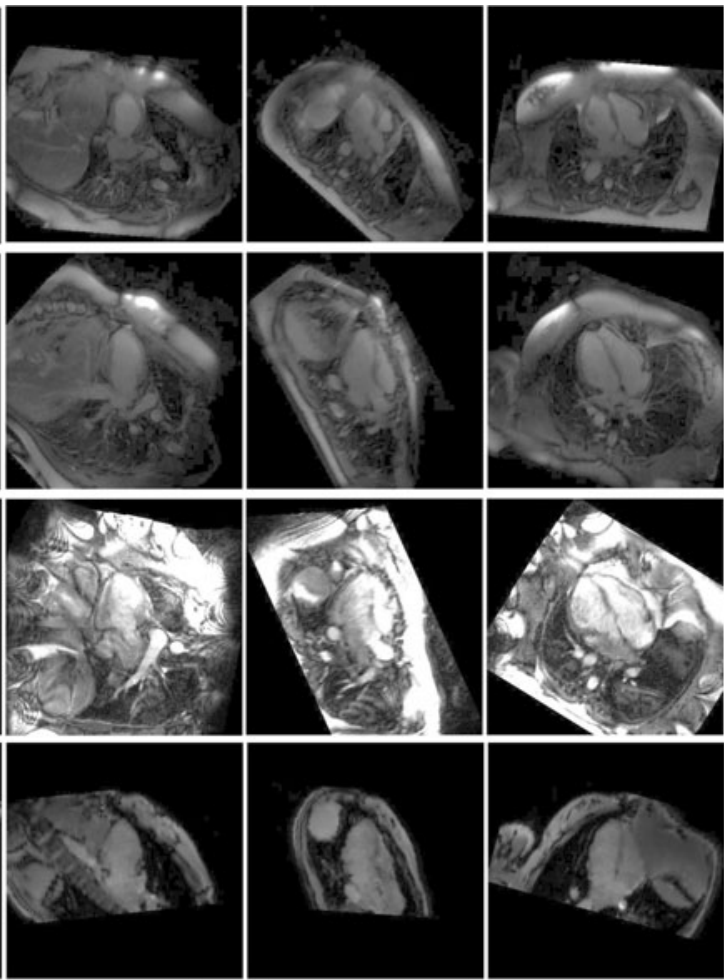

(c)

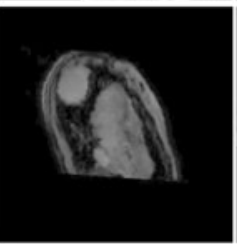

(d)

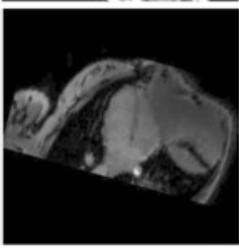

(e)

Fig. 4. Automatic view planning examples. (a) Original 3D volume. (b) LV delineation results. The prescribed 2-, 3-, and 4-chamber views are provided in (c)-(e), respectively.

Table 1. Automatic view planning evaluation by a 4 -fold cross validation. Axis and angle differences are measured in degrees and position difference is measured in $\mathrm{mm}$.

\begin{tabular}{|c|c|c|c|c|c|c|c|c|c|}
\hline & \multicolumn{3}{|c|}{ Short-axis stack } & \multicolumn{2}{|c|}{ 2-chamber view } & \multicolumn{3}{|c|}{ 3-chamber view } & \multicolumn{2}{|c|}{-chamber view } \\
\cline { 2 - 10 } & Axis & Base & Apex & Angle & Position & Angle & Position & Angle & Position \\
\hline Mean & 8.6 & 13.3 & 11.7 & 18.9 & 6.6 & 12.3 & 4.6 & 17.6 & 5.7 \\
\hline Std & 9.7 & 16.7 & 20.6 & 21.0 & 8.8 & 11.0 & 7.7 & 19.2 & 8.5 \\
\hline Median & 5.5 & 7.4 & 6.1 & 10.7 & 4.0 & 8.9 & 2.6 & 9.8 & 3.7 \\
\hline
\end{tabular}

\section{Conclusions}

We have presented a fully automatic and fast approach to view planning for cardiac MRI aquisition. Even in the presence of large image variations due to a mixture of experimental protocols, the proposed approach shows its capability to handle a majority of cases with reasonable image quality. With this approach, time for LV acquisition planning can be significantly reduced. Our future focus is to further improve view planning accuracies to overcome poor image quality and large image variation challenges. 


\section{References}

1. Cardiac MR Left Ventricle Segmentation Challenge. In: MICCAI Workshop (2009), http://smial.sri.utoronto.ca/LV_Challenge/Home.html

2. Attili, A.K., Schuster, A., Nagel, E., Reiber, J.H.C., van der Geest, R.J.: Quantification in cardiac MRI: advances in image acquisition and processing. Int. J. Cardiovasc Imaging 26, 27-40 (2010)

3. Cootes, T.F., Taylor, C.J., Cooper, D.H., Graham, J.: Active shape models - their training and application. Comp. Vision and Image Understanding 61, 38-59 (1995)

4. Danilouchkine, M.G., Westenberg, J.J.M., Reiber, J.H.C., Lelieveldt, B.P.F.: Accuracy of short-axis cardiac MRI automatically derived from scout acquisitions in free-breathing and breath-holding modes. Magnetic Resonance Materials in Physics, Biology and Medicine 18(1), 7-18 (2005)

5. Frangi, A., Niessen, W., Viergever, M.: Three-dimensional modeling for functional analysis of cardiac images: A review. IEEE Trans. on Medical Imaging 20(1) (2001)

6. Jackson, C.E., Robson, M.D., Francis, J.M., Noble, J.A.: Computerised planning of the acquisition of cardiac MR images. Computerized Medical Imaging and Graphics 28(7), 411-418 (2004)

7. Lelieveldt, B.P.F., van der Geest, R.J., Lamb, H.J., Kayser, H.W.M., Reiber, J.H.C.: Automated observer-independent acquisition of cardiac short-axis MR images: A pilot study. Radiology 221, 537-542 (2001)

8. Lelieveldt, B.P.F., Sonka, M., Bolinger, L., Scholz, T.D., Kayser, H., van der Geest, R., Reiber, J.H.C.: Anatomical modeling with fuzzy implicit surface templates: Application to automated localization of the heart and lungs in thoracic MR volumes. Computer Vision and Image Understanding 18(3), 218-230 (1999)

9. Lu, X., Georgescu, B., Jolly, M., Guehring, J., Young, A., Cowan, B., Littmann, A., Comaniciu, D.: Cardiac anchoring in mri through context modeling. In: Jiang, T., Navab, N., Pluim, J.P.W., Viergever, M.A. (eds.) MICCAI 2010. LNCS, vol. 6361, pp. 383-390. Springer, Heidelberg (2010)

10. Tu, Z.: Probabilistic boosting-tree: Learning discriminative models for classification, recognition, and clustering. In: Proc. ICCV, pp. 1589-1596 (2005)

11. Viola, P., Jones, M.J.: Robust real-time face detection. International Journal of Computer Vision 57(2), 137-154 (2004)

12. Zheng, Y., Barbu, A., Georgescu, B., Scheuering, M., Comaniciu, D.: Fast automatic heart chamber segmentation from 3D CT data using marginal space learning and steerable features. In: Proc. ICCV (2007) 